\title{
New approach to single-shot CARS thermometry of high-pressure, high-temperature hydrocarbon flames
}

\author{
V. I. Fabelinsky, ${ }^{1}$ V. V. Smirnov, ${ }^{1 *}$ O. M. Stel'makh, ${ }^{1}$ K. A. Vereschagin, ${ }^{1}$ A. K. Vereschagin, ${ }^{1}$ \\ W. Clauss ${ }^{2}$ and M. Oschwald ${ }^{2}$ \\ ${ }^{1}$ General Physics Institute, IOFAN, Vavilov Str. 38, 119991 Moscow, Russia \\ 2 German Aerospace Research Center, DLR, Langer Grund, D-74239 Hardthausen, Germany
}

Received 1 December 2006; Accepted 27 December 2006

\begin{abstract}
We demonstrate the feasibility of a proposed new approach to CARS thermometry with simultaneous measurements of the intensities of the hydrogen Q-branch lines and their linewidths during a single laser shot by means of a spectrograph combined with an interferometer having orthogonal directions of dispersions. This allows correct temperature evaluation that takes into account the directly measured linewidths. The experiments were carried out in a $\mathrm{H}_{2}: \mathrm{N}_{2}=1: 10$ mixture in a heated cell at a temperature of $1050 \mathrm{~K}$. The values of the gas mixture temperature, which were defined from dual-broad band (DBB) CARS spectra with linewidth corrections in a pressure range of 2-9 MPa, coincide with thermocouple measurements within $\sim 2 \%$, while without such a correction, the discrepancies increase up to $5 \%$. The proposed approach can be applied to CARS temperature measurements in investigations of $\mathrm{H}_{2}$ and hydrocarbon combustions without the necessity of species concentration measurements and not requiring the knowledge of line-broadening coefficients. Copyright @ 2007 John Wiley \& Sons, Ltd.
\end{abstract}

KEYWORDS: CARS spectroscopy; combustion thermometry; line broadening

\section{INTRODUCTION}

Optical diagnostics, in particular, coherent anti-Stokes Raman scattering (CARS) spectroscopy, because of its capability of nonintrusive measurements that allows quantitative temperature measurements and species concentration characterization, is a key methodology for combustion research. At present CARS is nearly the only optical diagnostic technique that allows obtaining quantitative data in the combustion chamber at pressures in the range from 1 to $10 \mathrm{MPa}$ and temperatures up to $3000 \mathrm{~K}$. Single-shot CARS thermometry can resolve spatial and temporal details of the combustion process and provide information on the combustion efficiency.

In case of CARS thermometry of $\mathrm{H}_{2} / \mathrm{O}_{2}$ flames, information on the gas temperature is derived from the CARS spectra of hydrogen probe molecules. For that, one has to know spectral line intensities and shapes, or widths. In the temperature range $1000-2700 \mathrm{~K}$, experimentally measured and extrapolated broadening coefficients of various $\mathrm{H}_{2} \mathrm{Q}$-branch lines perturbed by collisions with water molecules ${ }^{1-4}$ can be successfully used. For hydrocarbon (HC) fueled flames, the

*Correspondence to: V. V. Smirnov, General Physics Institute, IOFAN, Vavilov Str. 38, 119991 Moscow, Russia.

E-mail: vvs@kapella.gpi.ru problem of correct temperature evaluation still persists. This is mainly due to the lack of knowledge of $\mathrm{H}_{2}-\mathrm{CH}_{4}, \mathrm{H}_{2}-\mathrm{CO}$, $\mathrm{H}_{2}-\mathrm{CO}_{2}$, etc. collisional line-broadening coefficients. Additional experimental complications arise from the necessity of simultaneous measurements of $\mathrm{H}_{2} \mathrm{O}, \mathrm{CO}, \mathrm{CO}_{2}$, and $\mathrm{CH}_{4}$ concentrations during a single laser pulse. Moreover, for $\mathrm{H}_{2}$ / air and $\mathrm{HC} /$ air combustions at high temperatures and nitrogen concentrations, a specific collisional effect occurs for $\mathrm{H}_{2}-\mathrm{N}_{2}$ collision, leading to asymmetric line profiles and nonlinear dependence of the linewidth on the $\mathrm{H}_{2} / \mathrm{N}_{2}$ concentration ratio. This effect was observed and interpreted by using a model accounting for the speed dependence of the collisional line broadening and line shift. 5,6

To overcome this difficulty of gas thermometry as applied to $\mathrm{H}_{2}-\mathrm{OX}$ and $\mathrm{HC}-\mathrm{OX}$ combustion, a new experimental modification of CARS spectroscopy was proposed and tested. The idea lies in the simultaneous measurements of intensities of hydrogen Q-branch lines and their linewidths with the help of a spectrograph combined with an interferometer having orthogonal directions of dispersions. The experimental information on linewidths obtained from a single-shot CARS spectrum is directly used in temperature evaluation routines and does not require knowledge of species concentrations and density of the medium. 
Here we report the results of the application of this approach to single-shot CARS thermometry of a hydrogen: nitrogen $(1: 10)$ mixture in a heatable cell at high pressures (up to $9 \mathrm{MPa}$ ) and high temperatures ( $\sim 1000 \mathrm{~K}$ ), which was verified by thermocouple measurements. The main aim of this experiment was an investigation of the suggested technique from the sensitivity and reliability points of view in the course of intensity and linewidth measurements of the hydrogen Q-lines at high-temperature and high-pressure conditions.

\section{EXPERIMENTAL}

\section{Dual-broad band CARS spectrometer}

In our experiments, the dual-broadband (DBB) CARS technique is employed for rapid temperature measurements (during one laser shot). It should be noted that in case of hydrogen thermometry, DBB CARS, ${ }^{7}$ especially in conjunction with modeless lasers that eliminate the noise originating from mode competition, seems to be preferable to the conventional broadband CARS, ${ }^{4,8-10}$ since it provides the most efficient averaging over the spectra of the laser radiation, leading to the improvement of the quality of the CARS spectra and hence the precision of temperature measurements.

The layout of experimental setup is shown in Fig. 1. The laser part of our CARS spectrometer consists of a 'Quantel' $\mathrm{Nd}^{3+}$ : YAG laser delivering $100-200 \mathrm{~mJ}$ in the second harmonic beam and two broadband oscillator-amplifier dye lasers. Part of the $532 \mathrm{~nm}$ beam energy $(\sim 30 \mathrm{~mJ})$ was used as the CARS pump, the rest being used to pump the dye laser system. The second harmonic radiation had approximately $100 \mathrm{MHz}$ linewidth. DBB pumping was realized with the help of two oscillator-amplifier dye lasers. Dye laser I was a modeless Rhodamine $6 \mathrm{G}$ dye laser operating around $553 \mathrm{~nm}$ with a bandwidth of $\sim 170 \mathrm{~cm}^{-1}$ and output energy of about $3 \mathrm{~mJ}$. Dye laser II was a pyridine 1 / dimethyl sulfoxide (DMSO) laser with a glass substrate used as an output coupler of the oscillator. The dye laser II output power reached $1.5 \mathrm{~mJ}$, its lasing being centered at approximately $716 \mathrm{~nm}$ with a bandwidth $\sim 350 \mathrm{~cm}^{-1}$. The difference between the central wavenumbers of the two dye lasers was $\sim 4000 \mathrm{~cm}^{-1}$ and the width of the convolution of the spectra of these lasers allowed simultaneous recording of the $Q_{1}-Q_{7}$ lines of a hydrogen molecule. The pump beams, adjusted in diameters and divergences and with their pulses overlapped in time, were configured in the 'planar CARS' geometry. With the 300 -mm focal length focusing lens, the beam waist diameters in the focal region were estimated to be about $100 \mu \mathrm{m}$. The longitudinal spatial resolution for the employed interaction geometry, defined as the length of the volume in which over $90 \%$ of the CARS signal is generated, was measured to be $2.5 \mathrm{~mm}$

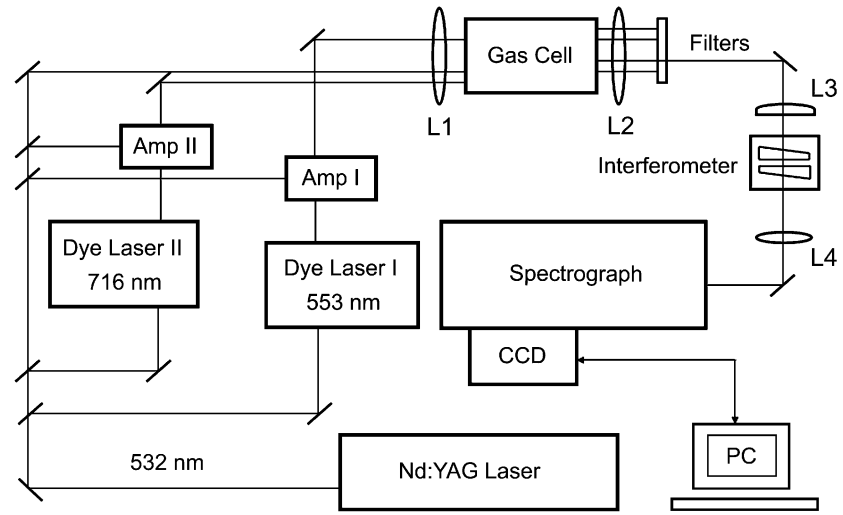

Figure 1. Layout of the experimental setup.

\section{CARS spectra detection system}

The analysis of the line shapes of individual hydrogen Q-branch lines with the necessary spectral resolution was performed with the help of a Fabry-Perot interferometer, which was placed (together with additional optical lenses) in front of the spectrograph. The dispersions of the interferometer and the spectrograph were in orthogonal directions (vertical and horizontal, respectively). The anti-Stokes beam going out from the cell, collimated by lens 2 and after preliminary filtration by dichroic and notch filters, was focused in a vertical direction onto the interferometer by the cylindrical lens 3. In the horizontal direction the beam remained collimated. Beams going out from the interferometer were focused with lens 4 onto the entrance slit of a grating spectrograph. As a result, a two-dimensional image was formed at the focal plane of the spectrograph with Q-branch lines plotted along a horizontal direction each having an interference pattern in the vertical direction (Fig. 2). This two-dimensional intensity distribution was detected by a gated microchannel plate (MCP) image intensifier CCD detector (Andor MCD).

A Jobin-Yvon HR500 spectrograph equipped with a 1200 grooves $/ \mathrm{mm}$ holographic grating, having a $1.2 \mathrm{~nm} / \mathrm{mm}$

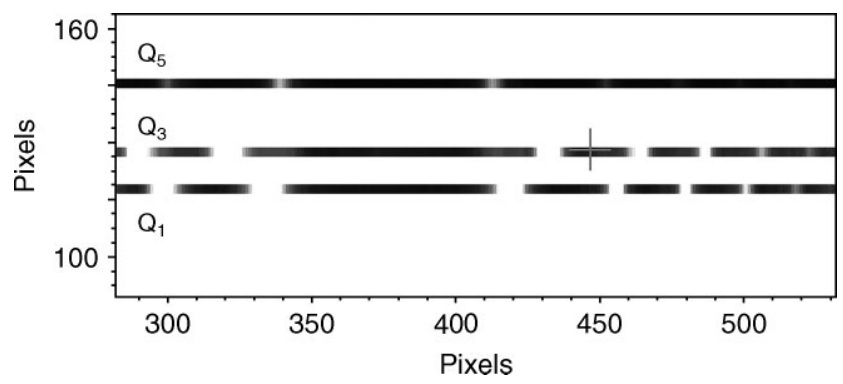

Figure 2. Image of the interferogram for the $Q_{1}, Q_{3}$, and $Q_{5}$ lines of hydrogen recorded during a single shot. The dispersion of the spectrograph is along the $Y$-axis. Three tracks with 3 pixels width for each correspond to the positions of maximum intensities of the Q-branch lines. The dispersion of the interferometer is along the $X$-axis. The center of symmetry of each interferogram is located at pixel number 375 . 
$\left(1.8 \mathrm{~cm}^{-1} /\right.$ pixels $)$ dispersion and approximately $15 \mathrm{~cm}^{-1}$ width of the slit function was sufficient to separate and resolve the $\mathrm{H}_{2}$ Q-branch lines.

In order to figure out the slit function of the interferometer, it was illuminated by the CARS light generated in a cell containing $0.1 \mathrm{MPa}$ of hydrogen at room temperature. In this case, the CARS spectrum has a linewidth of $\sim 0.017 \mathrm{~cm}^{-1}$ [half width at half-maximum (HWHM) Doppler linewidth at $300 \mathrm{~K}$. In our experiments, two different interferometers with mirror spacings of $1 \mathrm{~mm}$ or $3 \mathrm{~mm}$ and slit widths functions (HWHM) of $0.217 \mathrm{~cm}^{-1}$ and $0.067 \mathrm{~cm}^{-1}$, respectively, were used. These values of the slit functions were sufficient to determine the widths of Q-branch lines and were later taken into account while processing the linewidth data.

In order to reduce the readout time of the CCD and to provide simultaneous detection of $\mathrm{Q}_{1}, \mathrm{Q}_{3}$, and $\mathrm{Q}_{5}$, three corresponding CCD tracks along the direction perpendicular to the spectrograph dispersion were chosen (Fig. 2). The width of each track was equal to 3 pixels and their centers coincided with the positions of the Q-branch lines.

\section{The high-pressure heatable cell}

Our experiments were carried out in a heatable high-pressure cell with a very weak gas flow of a $1: 10 \mathrm{H}_{2}: \mathrm{N}_{2}$ mixture. The mixture was prepared well in advance. Pressure in the cell could be changed in the range from 0.1 to $10 \mathrm{MPa}$. The precision of pressure measurement was $\pm 0.01 \mathrm{MPa}$. The $100-\mathrm{mm}$ long and 12-mm diameter cell could be heated by an electric heater up to the temperature of $1100 \mathrm{~K}$. The temperature in the cell was measured by a thermocouple with a precision of $\pm 1^{\circ} \mathrm{C}$. In order to avoid the influence of temperature gradients on the results of our experiments, the thermocouple was located only a few millimeters above the CARS beam waists.

\section{RESULTS AND DISCUSSION}

When performing CARS diagnostics of nonstationary flames, it is necessary to record the spectra during a single laser shot. Usually this is performed with the help of a moderate-resolution grating spectrograph having a slit function much broader than the widths of the molecular lines themselves. Flame temperature is then evaluated by fitting the experimental moderate-resolution spectra of the probe molecule to the simulated ones, which in turn are calculated as a convolution of the spectral profile of a nonlinear susceptibility $\left|\chi^{(3)}\right|^{2}$ with the slit function $\psi(\omega)$ of a spectrograph. If homogeneous collisional line broadening takes place when calculating $\left|\chi^{(3)}\right|^{2}$ hydrogen spectra of Q-branch transitions, a superposition of Lorentzian lines can be used. After the convolution, the resulting CARS spectrum of $\mathrm{H}_{2}$ Q-branch will be presented as a sum of spectrograph slit functions, each component having an amplitude proportional to the integral intensity of a real
(Lorentzian) hydrogen line:

$$
\begin{aligned}
& I_{\text {CARS }}(\omega)=\sum_{j} I_{\Sigma}(j) \times \int_{-\infty}^{\infty} \delta\left(\xi-\omega_{0 j}\right) \times \Psi(\omega) \mathrm{d} \xi \\
& I_{\Sigma}(j) \sim \int_{-\infty}^{\infty} \frac{\left(N_{v=0, j}-N_{v=1, j}\right)^{2}}{\left(\omega-\omega_{0 j}\right)^{2}+\Gamma_{j}^{2}} \mathrm{~d} \omega \sim \frac{\left(N_{v=0, j}-N_{v=1, j}\right)^{2}}{\Gamma_{j}}
\end{aligned}
$$

Hence, knowledge of the widths of the hydrogen lines together with their concentration, temperature, and $J$-dependence is required for correct calculation and interpretation of broadband CARS spectra. In a multicomponent mixture, information on line-broadening coefficients of the probe molecule by all other molecules and also on all species concentrations is necessary.

Hydrogen-oxygen flames contain the smallest number of the main line-broadening species: hydrogen, oxygen, and water. The hydrogen lines contour, perturbed by $\mathrm{H}_{2}-\mathrm{H}_{2}$ and $\mathrm{H}_{2}-\mathrm{H}_{2} \mathrm{O}$ collisions at high pressures and temperatures, is broadened homogeneously and has Lorentzian shape, and the line-broadening coefficients together with their $J$ - and temperature dependence are known. ${ }^{1-4,11}$ In this case, a temperature evaluation from a single-shot CARS spectrum can be performed if the problem of determination of species concentration is solved.

In case of temperature measurements in $\mathrm{H}_{2}$-air flames, the situation becomes more complex because, in addition to the above-mentioned collisions, there exist collisions of $\mathrm{H}_{2}$ with $\mathrm{N}_{2}, \mathrm{CO}_{2}$, and $\mathrm{CO}$, which can alter the hydrogen line shape in a significantly different way. As already mentioned, the hydrogen line contour perturbed by collisions with nitrogen contains both homogeneous and inhomogeneous contributions, the latter due to a speed-dependence effect. The lineshape becomes asymmetrical and deviates from a Lorentzian. The authors of Refs 5 and 6 developed models of collisional broadening that take into account the speeddependence effect, which allowed them to describe the lineshape and its peculiarities depending on temperature and $\mathrm{H}_{2} / \mathrm{N}_{2}$ concentration. In Ref. 12 a detailed analysis of the temperature evaluation problem in a hydrogen-nitrogen mixture has been performed with the help of the results obtained in Ref. 6. It was shown that the use of a Lorentzian profile instead of an asymmetrical theoretical model profile with equal width expressed by a polynomial law to represent concentration and temperature functional dependencies allows significant simplification of calculations without essential loss of temperature determination accuracy $(<3 \%)$.

In the approach suggested in our work, the linewidths are measured simultaneously with CARS intensities. That allows correct temperature evaluation during a single laser shot without the necessity of concentration measurements and not requiring the knowledge of the complete set of line-broadening coefficients.

All experiments were carried out in $\mathrm{a}_{2}: \mathrm{N}_{2}=1: 10$ mixture at a cell temperature of $1050 \mathrm{~K}$. The optical system has been adjusted to form symmetrical illumination of 
the interferometer by the CARS beam in order to avoid distortions of the interference pattern images on the CCD detector. The resulting interferogram image (Fig. 2) and its corresponding 3D graphical representation (Fig. 3) obtained at $0.5 \mathrm{MPa}$ mixture pressure and $1050 \mathrm{~K}$ cell temperature show good signal-to-noise ratio which seems to be sufficient for temperature measurements in real combustions. Similar spectra of Q-branch lines with $J=1,3$, and 5 were recorded in the pressure range of 1-9 MPa. Following the idea developed in Ref. 12, we have used the Lorenzian profiles in the fitting procedure of the experimental interferograms to deduce the linewidths of the $Q_{1}, Q_{3}$, and $Q_{5}$ lines. Figure 4 presents the result of such a procedure for the interferogram of $Q_{3}$ line recorded at 9 MPa mixture pressure. All the widths of the $Q_{1}$, $\mathrm{Q}_{3}$, and $\mathrm{Q}_{5}$ lines measured in the pressure range 1-9 MPa at $1050 \mathrm{~K}$ in a $10 \% \mathrm{H}_{2}+\mathrm{N}_{2}$ mixture are well described by linear dependencies of linewidth versus the total mixture pressure. The corresponding line-broadening coefficients $Y$ (HWHM) are presented in Fig. 5, which demonstrates good agreement with the data from Ref. 6 for the $Q_{1}$ line.

Taking into account the information on linewidths, the temperature evaluation can be provided using either peak or integral intensities of the Q-branch lines (Fig. 3).

The integral intensities of the hydrogen $Q_{1}, Q_{3}$, and $\mathrm{Q}_{5}$ lines were obtained by integrating the interferograms over either one or several orders (see Figs 2 and 3) along the direction of dispersion of the interferometer. As the Qbranch lines have different wavelengths, the interference patterns are situated at different positions relative to the plane of symmetry of the interferogram, and their intensities were normalized on the spatial profile of the anti-Stokes beam intensity (long dashed line in Fig. 4). It should be pointed out that these values of integral intensities are proportional to the maximum values of integral intensities in the profiles of conventional DBB CARS. ${ }^{1,2}$ For illustration, in Fig. 6 the image and the intensity profile of the hydrogen Qbranch DBB CARS spectra recorded with the interferometer removed are presented. Further, for temperature evaluation, the obtained integral intensities of the Q-branch lines were

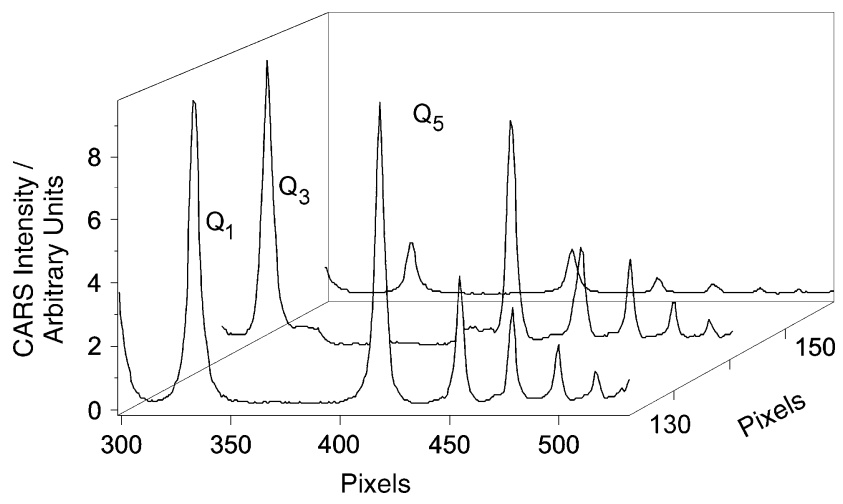

Figure 3. 3D presentation of intensities profiles of the interferograms for the $Q_{1}, Q_{3}$, and $Q_{5}$ lines recorded on the base of an image (see Fig. 2).

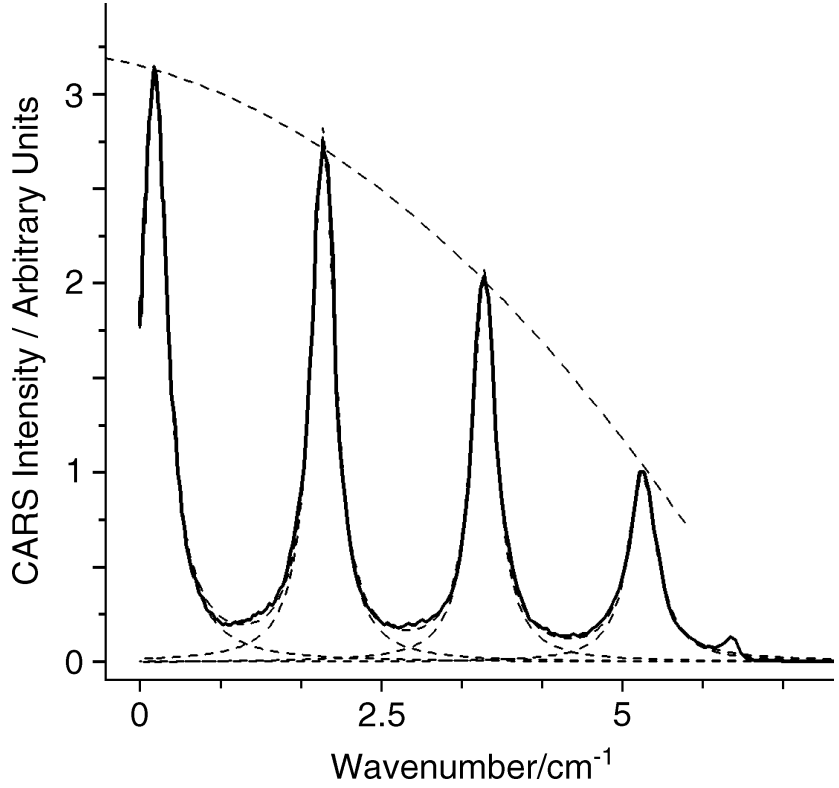

Figure 4. The intensity profile of the right part of the interferogram for the $Q_{3}$ line obtained at a pressure of the gas mixture of $9 \mathrm{MPa}$ and presented in a linearized scale. Solid line-experimental curve. Short dash-result of fitting by Lorentzian profiles. Long dash-profile of anti-Stokes beam intensity.

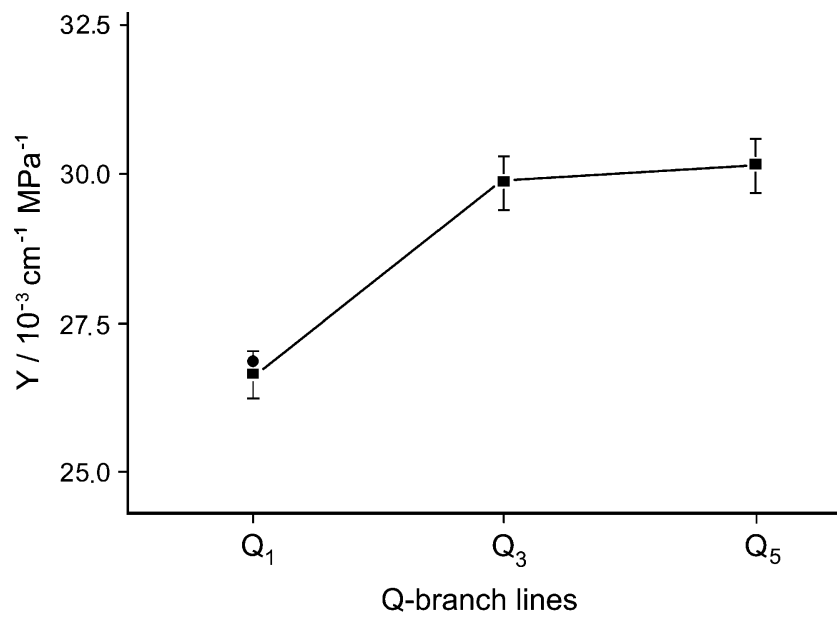

Figure 5. The broadening coefficients $Y$ for the hydrogen Q-branch lines in a mixture with nitrogen $\mathrm{H}_{2}: \mathrm{N}_{2}=1: 10$ at temperature $T=1050 \mathrm{~K}$. ๑: data taken from Ref. 6 . $\mathbf{\square}$ : this work.

normalized on the profile of the nonresonant DBB CARS spectrum recorded at $1 \mathrm{MPa}$ of pure $\mathrm{N}_{2}$. Taking into account the measured values of the integral intensities and the linewidth $\Gamma$ for $Q_{1}, Q_{3}$, and $Q_{5}$ lines, we evaluated the temperature as a parameter of the Boltzman distribution of the population of the rotational levels.

The temperature values of the gas mixture in a pressure range of 2-9 MPa defined from DBB CARS spectra according 


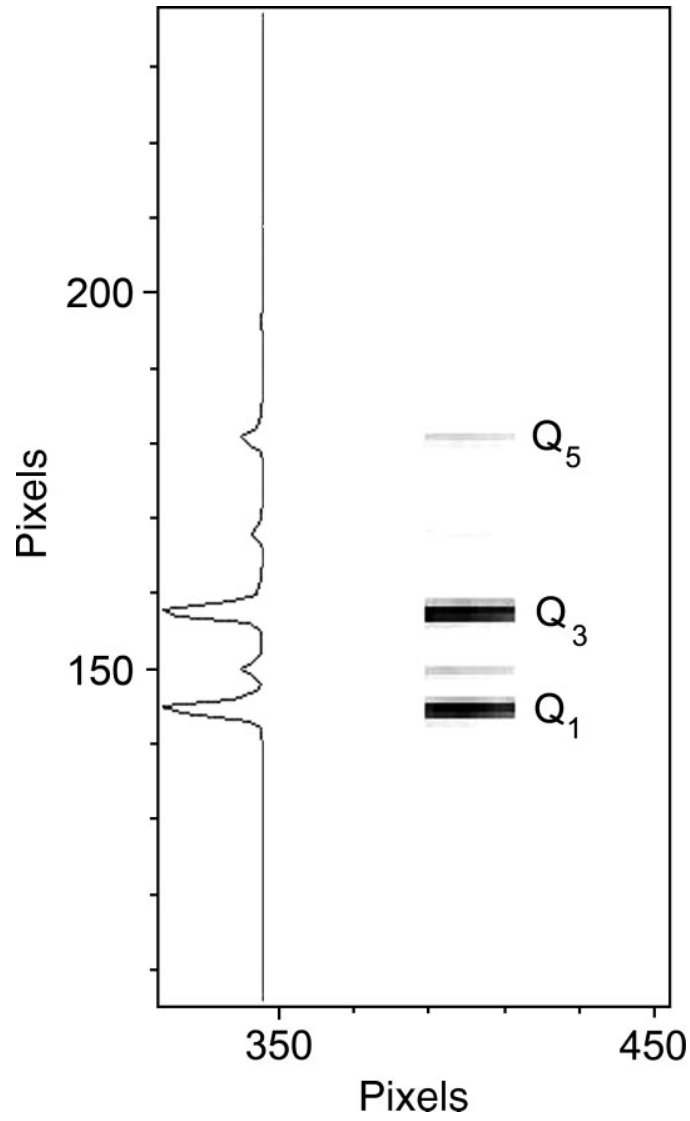

Figure 6. The image and intensity profiles of DBB CARS spectra of the hydrogen Q-branch recorded with the interferometer removed.

to procedure described above with linewidth corrections coincide with thermocouple measurements within $\sim 2 \%$ (Fig. 7), while without such a correction the discrepancies increase up to $5 \%$. At low pressures (below $2 \mathrm{MPa}$ ), this discrepancy decreases because of the transition from homogeneously broadened lines exhibiting $J$-dependence of the linewidths to inhomogeneously (Doppler) broadened lines all having equal widths.

\section{CONCLUSIONS}

We have demonstrated the success of a proposed approach to single-shot DBB CARS temperature measurements in a hydrogen-nitrogen mixture in which the hydrogen line shape is perturbed by a speed-dependence effect-the case that is the most doubtful from the point of view of describing the spectrum of nonlinear susceptibility by the sum of Lorentzian profiles. It should be noted that $\mathrm{H}_{2}+\mathrm{O}_{2}$ or $\mathrm{HC}+\mathrm{O}_{2}$ combustion mixtures contain water vapor as a main product and in this case the $\mathrm{H}_{2}$ lines demonstrate homogeneous broadening with pure Lorentzian profiles caused by collisions with water molecules. ${ }^{13}$ This means that the proposed approach can be employed for

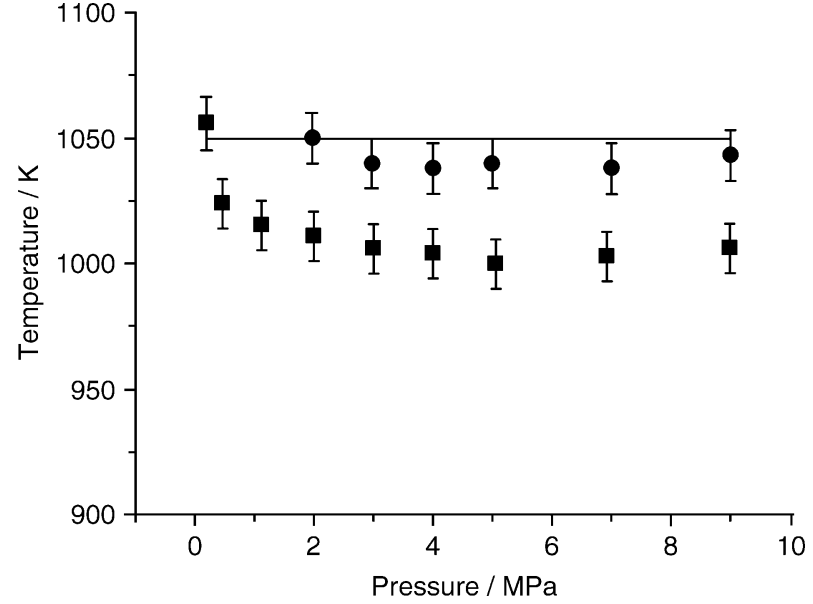

Figure 7. The temperature of the gas mixture $\mathrm{H}_{2}: \mathrm{N}_{2}=1: 10$ in the heatable high-pressure cell. Solid line - thermocouple measurement $T=1050 \mathrm{~K}$. The values of temperature were measured by DBB CARS at different pressures of the gas mixture: $\mathbf{\square}$ : results of temperature evaluation without correction for linewidths. @: with linewidth correction.

temperature measurements in combustion investigations of the $\mathrm{H}_{2} / \mathrm{HC}+$ air as well as $\mathrm{H}_{2} / \mathrm{HC}+\mathrm{GOX}$ (gaseous oxygen) flames.

\section{Acknowledgements}

The authors gratefully acknowledge financial support for parts of this work by INTAS (project No. INTAS 05-1000008-7951. Field 6).

\section{REFERENCES}

1. Berger J-P. Thèse de Doctorat. Université de Bourgogne, Dijon, 1994.

2. Michaut X, Berger J-P, Saint-Loup R, Chaussard F, Berger H. Combustion dans les Moteurs Fusées, 26-28 June 2001, Actes $d u$ Collogue de Synthèse $d u$ Groupe de Recherche CNES/CNRS/ONERA/SNEGMA. Cepadues Editions: Toulouse, $2001 ; 321$.

3. Shaussard F, Michaut X, Saint-Loup R, Berger H, Joubert P, Lance B, Bonamy J, Robert D. J. Chem. Phys. 2000; 112: 158

4. Clauss W, Klimenko DN, Oschwald M, Vereschagin KA, Smirnov VV, Stelmakh OM, Fabelinsky VI. J. Raman Spectrosc. 2002; 33: 906.

5. Farrow RL, Rahn LA, Sitz GO, Rosasko GJ. Phys. Rev. Lett. 1989; 63: 746.

6. Sinclair PM, Berger JPh, Michaut X, Saint-Loup R, Chaux R, Berger H, Bonamy J, Robert D. Phys. Rev., A 1996; 54: 402.

7. Vereschagin KA, Smirnov VV, Stel'makh OM, Fabelinsky VI, Clauss W, Klimenko DN, Oschwald M, Vereschagin AK. J. Raman Spectrosc. 2005; 36: 134.

8. Bergmann V, Stricker W. Appl. Phys. B 1995; 61: 49.

9. Hancock RD, Bertagnolli KE, Lucht RP. Combust. Flame 1997; 109: 323.

10. Stricker W, Woyde M, Luckerath R, Bergmann V. Ber. BunsenGes. Phys. Chem. 1993; 97: 1608.

11. Rahn LA, Farrow RL, Rosasco GJ. Phys. Rev., A 1991; 43: 6075.

12. Hussong J, Stricker $W$, Bruet $X$, Joubert $P$, Bonamy J, Robert $D$, Michaut X, Gabard T, Berger H. Appl. Phys. B 2000; 70: 447.

13. Hussong J, Lückerath $R$, Stricker $W$, Bruet $X$, Joubert $P$, Bonamy J, Robert D. Appl. Phys. B 2001; 73: 165. 